\title{
Einige fragmentarische Grabinschriften aus dem antiken Kurort Allianoi
}

\author{
Antik Sağlık Merkezi Allianoi’dan birkaç Mezar Yazıtı Fragmanı
}

\begin{abstract}
Ahmet YARAŞ ${ }^{*}$
Ferit BAZ $^{* *}$

Zusammenfassung: Im Folgenden werden vier neue Inschriften aus dem antiken Kurort Allianoi behandelt, welche während der dortigen Notgrabungen zwischen den Jahren 1998 und 2006 aufgefunden und ins Pergamon Museum verbracht wurden. Die Inschriften sind sehr schlecht erhalten, vermutlich gehören sie verschiedenen Grabmonumenten. Bei der ersten Inschrift kommt ein Grab für eine Person namens Billas in Frage. In der zweiten Inschrift handelt es sich um ein, von einer Person namens Glykon dediziertes Grab. Die dritte Inschrift zeugt für ein Grab, das vermutlich der Frau einer Person namens Hymnos gehört. Letzte Inschrift ist ein nicht näher einzuordnendes fragmentarisches Dokument, das eine Person mit dem Gentilnomen Flavia im Dativ erwähnt.
\end{abstract}

Schlüsselwörter: Allianoi, Kurort, Grabmonument, Nekropole

Özet: Makalede, 1998-2006 seneleri arasında Allianoi sağlık merkezinde yürütülen kurtarma kazıları sırasında bulunmuş ve sonradan Bergama Müzesi'ne getirilmiş olan dört adet yazıt ele alınmaktadır. Olasılıkla farklı mezar anıtlarına ait olan bu yazıtlar, günümüze oldukça kötü bir şekilde ulaşmışlardır. İlk yazıtta olasılıkla Billas adındaki bir kimsenin mezarı; ikinci yazıtta ise Glykon adındaki birisi tarafından adanmış bir mezar söz konusudur. Üçüncü yazıt, Hymnos adındaki bir kimsenin adı günümüze ulaşmamış olan karısına yaptırdığı mezarı belgelemektedir. Dördüncü ve son yazıtın işlevi belirlenememekle birlikte, bu yazıtta Flavia gens'ine mensup bir kadının ismi Dativus halinde zikredilmektedir.

Anahtar Kelimeler: Allianoi, Sağlık Merkezi, Mezar Anıtı, Nekropolis

Im Folgenden werden vier Inschriften aus der antiken Siedlung Allianoi behandelt, die während der dortigen Notgrabungen zwischen den Jahren 1998 und 2006 aufgefunden und ins Pergamon Museum verbracht wurden. Der Fundort Allianoi wurde von der Ausgrabungsmannschaft auf dem Landweg zwischen Bergama-Ivrindi 18 km nordöstlich lokalisiert (Müler, 2004, 215-225; Yaraş, 2006, 19-36; Yaraş, \& Erten, 2008, 83-91). Dieser Ort ist bis zum Jahre 2000 als Paşa Ilicası bekannt, der sich in der Mitte des Stausees des Yortanlı Staudamms befindet, der zum Bewässerungszwecken gebaut wurde. Auf unseren Vorschlag hin ist der antike Ort im Jahr 2001 als schützenswertes Bodendenkmal vom türkischen Kulturministerium ausgewiesen. Notgrabungen mit beschränkten Finanzmöglichkeiten wurden während lediglich neun Grabungssaisons durchgeführt, wodurch knapp \% 20 Prozent der gesamten Fläche der antiken Siedlung Allianoi ausgegraben werden konnten (siehe Abb. 1). Da die Forschungsgenehmigung seit 2007 nicht mehr erteilt wird, scheint es jetzt nicht mehr möglich zu sein, die ganze Siedlung auszugraben, d.h. die wissenschaftliche archäologische Arbeit wird nicht mehr zu Ende gebracht. In Folge dessen wird die weitere archäologische und epigraphische Erkenntnissgewinnung durch den

\footnotetext{
* Doç. Dr., Trakya Üniversitesi, Edebiyat Fakültesi, Arkeoloji Bölümü, Edirne, ahmetyaras@ hotmail.com

** Yrd. Doç. Dr., Mimar Sinan Güzel Sanatlar Üniversitesi, Tarih Bölümü, İstanbul, feritbaz@yahoo.com
} 
Bau dieses Staudamms unmöglich gemacht.

Dass die Geschichte der antiken Siedlung auf späthellenistische Zeit zurückgeht und eine rege Bautätigkeit dort in der römischen Kaiserzeit besonders im 2. Jh. n. Chr. unternommen wurde, ergaben die Notgrabungen. Außerdem stellte es sich heraus, dass Allianoi mit Sicherheit als zweites Heilzentrum neben Pergamon benutzt wurde (Yaraş, 2004-2005, 71-74; 2006, 453462). Trotz mehreren Naturkatastrophen wurde das Gebiet bis zur osmanischen Herrschaft kontinuierlich bewohnt, in dem sich in byzantinischer Zeit die Werkstätten für die Herstellung von Glass, Keramik und Eisen befanden. In der näheren Umgebung von Allianoi wurden das Vorhandensein einer römischen Straße zwischen Pergamon und Allianoi, römische Bauernhöfe, byzantinische Überreste und sechs Nekropolenbereiche nachgewiesen (Yaraş, 2004, 227-235).

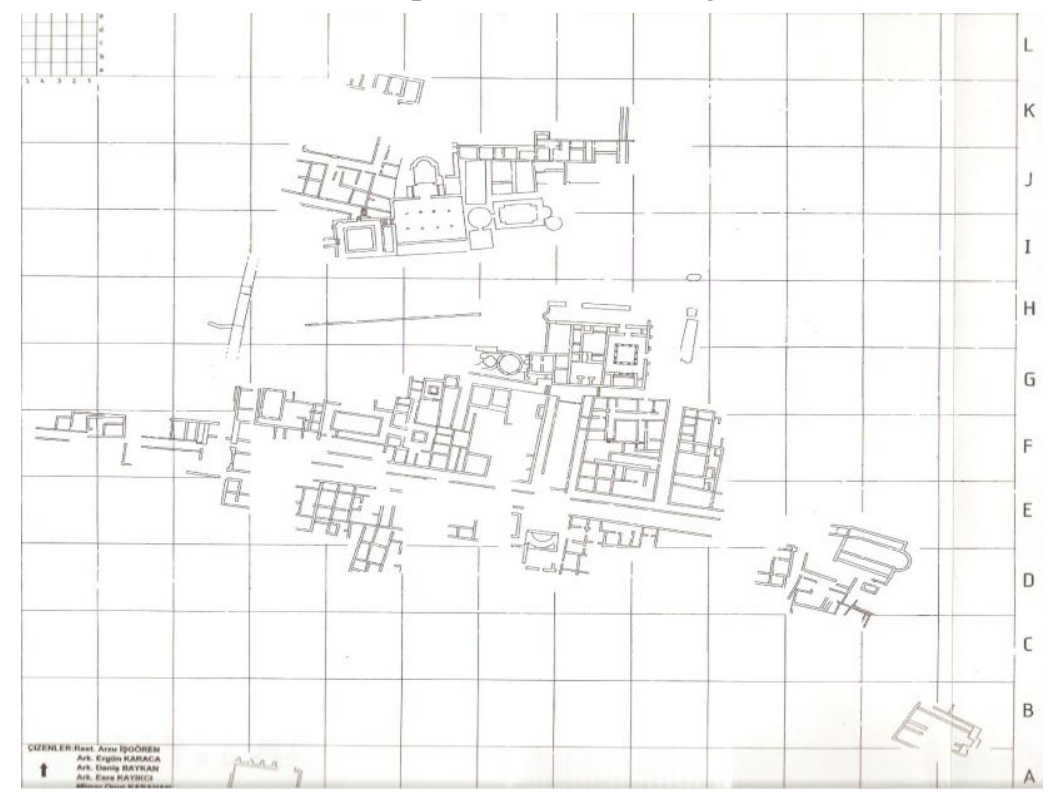

Abb. 1. Plan von Allianoi

\section{1) Vermutlich ein Grabmonument für Billas}

Ein fragmentarisches Stück aus Marmor mit Relief. Es wurde an der südwestlichen Ecke eines Ortes gefunden, wo sich Cardo und Decumanus im Kurort kreuzten EVIIIe5 (siehe Abb. 1). Der Inschriftenträger wurde später als Füllmaterial aus seinem unbekannten ursprünglichen Ort hierhin verbracht. Er besitzt eine Höhe von 18,3 cm, eine Breite von 15,6 cm und eine Tiefe von $7 \mathrm{~cm}$. der obere und linke Teil sind abgebrochen. Im erhaltenen Teil des Reliefs sind vordere Seite eines Pferdes und ein Altar auf der rechten Seite zu sehen (siehe Abb. 2). Die Fläche des Reliefs, welches unten durch ein Profil abgeschlossen ist, ist stark verwaschen. Die Inschrift von den zwei Zeilen ist auf dem Altar gesetzt, der unten und oben profiliert ist und eine Höhe von $11.4 \mathrm{~cm}$ und eine Breite $9.5 \mathrm{~cm}$ besitzt. Inventarnummer der Notgrabung ist T. 99/84 und Nummer des Museums ist 17.3.16.00.

Bí $\lambda \lambda a \nu-$ TOS

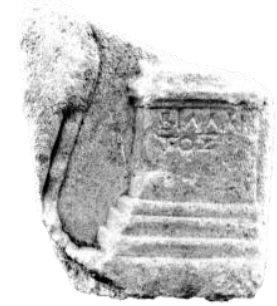

Abb. 2 
Bei oben vorgelegten Dokument handelt es sich vermutlich um ein Grabmonument für eine Person namens Billas. Es ist möglich, dass ein Reiter auf dem Pferd dargestellt wurde, wodurch der Verstorbene Billas als ein Reiter bezeichnet wurde. Der Name Billas scheint ein griechischer Name gewesen zu sein (Sundwall, 1913, 61; Robert, 1963, 16-18; Zgusta, 1964, 681. Zu den ähnlichen Namen wie Billos, Bilos, siehe Fraser, \& Matthews, 2000, 86; SEG 51, 2172; SEG 57, 1962). Es ist nicht ausgeschlossen, dass die Inschrift sich oberhalb oder unterhalb des Reliefs fortgesetzt hat und dadurch der Name der Dedikanten angegeben werden konnte (siehe etwa Pfuhl, \& Möbius, 1979, Tafel 190 no. 1282; Tafel 191 no. 1294). Das fragmentarische Relief des Monuments mit einem Pferd und einem Altar ist mit den einigen anderen Monumenten aus der Antike zu vergleichen (siehe Mitropoulou, 1975, no. 1, 9; Pfuhl, \& Möbius, 1979, Tafel 193-195 und 197-199; Atalay, 1988, no. 43; 47; Cremer, 1991, Tafel 8). Dass der Verfasser die Fläche des Altars mit der Schrift ausgefüllt hat, kann auch in einigen anderen Monumenten ebenfalls beobachtet werden ( $\mathrm{Zu}$ den Inschriften auf den Statuenbasen und Postamenten im Relief siehe etwa Pfuhl, \& Möbius, 1977, Tafel 96 no. 628; 1979, Tafel 300 no. 348$)$.

\section{2) Von Glykon dedizierte Grabstele}

Eine Stele aus Marmor, deren rechte Seite und unterer Teil abgebrochen sind. Sie wurde in dem Bereich FIX d1, wo sich westliche Stoa des Decumanus befindet, aufgefunden (siehe Abb. 1). Der Inschriftenträger scheint vermutlich zu den späteren Zeiten als Füllmaterial Anwendung gefunden zu haben. Er besitzt eine Höhe von $17.5 \mathrm{~cm}$, eine Breite von $14 \mathrm{~cm}$ und eine Tiefe von $5.6 \mathrm{~cm}$. Ihre Vorderseite ist mit einem Reiterrelief verziert, über welchen erste zwei Zeilen der Inschrift versetzt sind, während ihre Rückseite grob gelassen ist. Im Relief wurde ein Reiter, dessen Gesicht stark abgenutzt ist, auf einem Pferde dargestellt. Eine weite Pelerine bedeckt den Rücken des Reiters, welcher in seiner rechten Hand eine lange Lanze und in seiner linken Hand eine Schild hält (siehe Abb. 3). Das Schriftbild ist sorgfältig: Zwar besitzen die Buchstaben unterschiedliche Größen, sie sind aber in gleichen Abständen relativ tief eingraviert. Die Buchstabenhöhe ist $1 \mathrm{~cm}$. Inventarnummer der Notgrabung ist T.98/131 und Inventarnummer des Museums ist 22.15.00.

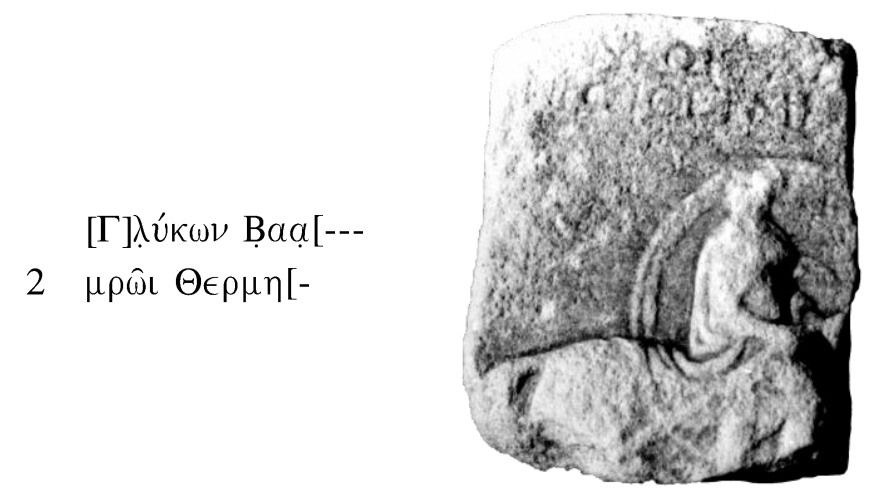

Abb. 3

Z. 1: Der zweite Name ist aller Wahrscheinlichkeit nach als der Name im Genitiv wie Baakov, Baavov vorzustellen (Zum Namen Baakos siehe MAMA VII 24; für Baanes siehe SEG 51, 1447).

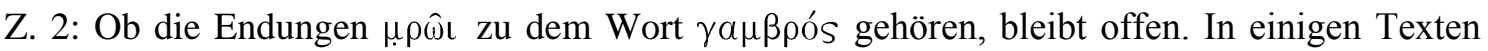

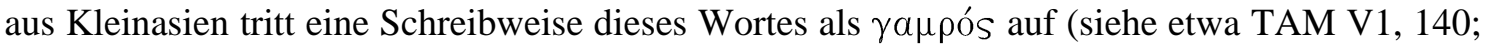
CIG III 4006).

Z. 2: Es liegt nahe, dass die Buchstaben $\Theta \epsilon \rho \mu \eta$ zu dem Begriff $\Theta \epsilon \rho \mu \eta \nu o ́ s$ gehören können, 
weil dieser Begriff in den epigraphischen Zeugnissen der antiken Kurbaden in Kleinasien oft Anwendung fanden (Liddell, \& Scott, 1968, 71; zu den epigraphischen Belegen des Begriffes als Ortsname siehe MAMA X 443; Schwertheim, 1987, no. 76. Ferner zur Gottheit Meter Thermene siehe Robert, 1955, 78-82) und die Siedlung Allianoi ebenfalls zu den antiken kleinasiatischen Heilthermen zählte (Allgemein für die Thermen in Kleinasien siehe besonders Debord, 1982, 28-33; Yaraş, 2004-2005, 71-74. Schwertheim, 1987, 58; Yegül, 1995, 92-127. Für eine solche Siedlung in Yalova in Bithynien siehe Mansel, 1936, 54-56; Corsten, 1987, 140-147; Günther, 1998, 105-117; Akyol, 2003, 27-28).

Der fragmentarische Erhaltungszustand lässt eine exakte Bestimmung des Charakters des Textes nicht mehr zu. Mit Sicherheit setzte sich diese fragmentarische Inschrift auf der rechten Seite und vermutlich im unteren Teil des Inschriftenträgers fort. Diese Inschrift gehört vermutlich zu einem Grabmonument. Hinsichtlich seiner Reiterdarstellung ist das Monument mit einigen antiken Stelen zu vergleichen (siehe beispielsweise Pfuhl, \& Möbius, 1979, Tafel 190-193), das von einem bestimmten Glykon dediziert wurde. Der griechische Name Glykon ist besonders im westlichen Kleinasien häufig bezeugt (Börker, \& Merkelbach, 1979, no. 552; Petzl, 1987, no. 752; Lajtar, \& Petzl, 2003, 46; 47; Malay, 2003, 13-18; Adak, \& Akyürek-Şahin, 2005, no. 24; Mägele, 2005, 291-292; Hermann, \& Malay, 2007, no. 70). In der Antike war der bekannteste Namensträger sicherlich die Gottheit Glykon, die als Reinkarnation von Asklepios betrachtet wurde (Meyer, 1987, 43-45; Marek, 2010, 648). Aus diesem Grund scheint es nicht verwunderlich zu sein, diesem Personennamen auf einem epigraphischen Monument des Kurortes Allianoi zu begegnen.

\section{3) Von Hymnos dediziertes Grabmonument}

Ein Block aus Marmor. Er wurde in dem Bereich DV b2, wo sich eine byzantinische Eisenwerkstatt befindet, aufgefunden (siehe Abb. 1). Der unterer Teil und die rechte Seite des Inschriftenträgers sind abgebrochen (siehe Abb. 4). Er besitzt eine Höhe von 9, $1 \mathrm{~cm}$, eine Breite von 10, $7 \mathrm{~cm}$ und eine Tiefe von 3,2 cm. Die Zeilen der Schrift sind geradlinig, die Buchstaben in unterschiedlichen Größen und in gleichen Abständen sind dünn eingemeißelt. Die Buchstabenhöhe schwankt zwischen 0, 9 und 2, $5 \mathrm{~cm}$. Inventarnummer der Notgrabung ist T.05/8.

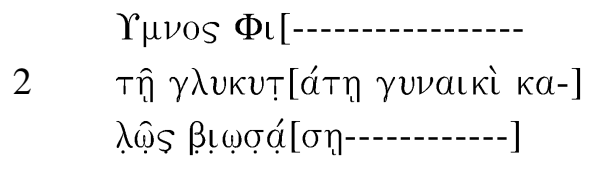

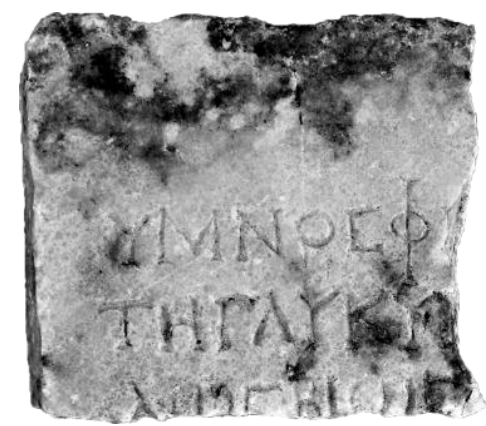

Abb. 4

Der Name des Dedikanten, Hymnos, ist in weiteren westlichen Städten des Kleinasiens bezeugt (siehe Petzl, 1982, no. 476; İçten, \& Engelmann, 1998, no. 3; Engelmann, 2000, no. 16). Die

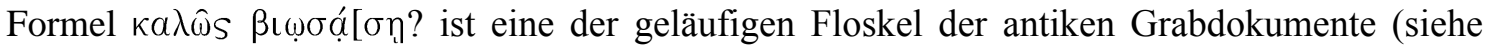
TAM V,1 122; 191; Studia Pontica III 159).

4) Eine nicht näher einzuordnende fragmentarische Inschrift (vermutlich ein Grabmonument für Flavia ...)

Ein Marmorfragment, welches in der mittleren Mauer von GI Latrina, südlich von Ilıca (GVIII 
c5), aufgefunden wurde (siehe Abb. 1). Der Inschriftenträger ist allseitig abgebrochen (siehe Abb. 5). Er besitzt eine Höhe von 16, $7 \mathrm{~cm}$., eine Breite von $26 \mathrm{~cm}$ und eine Tiefe von 2, $5 \mathrm{~cm}$. Auf die Schrift des Fragments wurde nur geringe Sorgfalt verwandt; seine nach links geneigten Buchstaben besitzen unterschiedliche Größe und Abstände. Buchstabenhöhe schwankt zwischen 2, 2 und 5, $3 \mathrm{~cm}$. Inventarnummer der Notgrabung ist T.04/ 3 .

$$
\begin{aligned}
& \text { E } \sum \sum T A[ \\
& \text { Tฺ̂ } \Phi \lambda \text {. \$ ? } \\
& \mathrm{AI} \Omega \mathrm{N}[---
\end{aligned}
$$

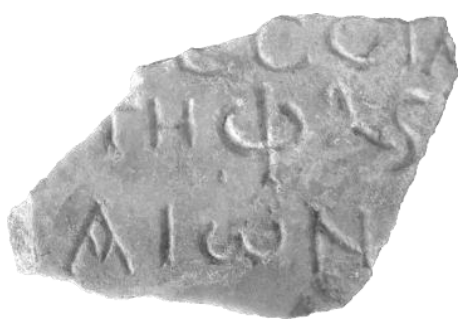

Abb. 5

Aufgrund des schlechten Erhaltungszustands scheint Anordnung des Dokuments nicht möglich zu sein. Vermutlich kommt hier eine Dedizierung eines antiken Objekts (Grab?) für eine Frau mit römischem Bürgerrecht in Frage. Sie oder einer ihrer Vorfahren könnte römisches Bürgerrecht in flavischer Zeit erworben haben.

In der Spätantike müssen die oben vorgelegten fragmentarischen Inschriften zu den verschiedenen Grabmonumenten als Spolien vermutlich aus dem sogenannten Devlet Orman1 Nekropolenbereich, westlich von Allianoi gelegen, zu den oben erwähnten jeweiligen Fundorten verbracht gewesen sein. So gesehen stammen sie mit größter Wahrscheinlichkeit aus diesem Nekropolenbereich, der einer von den nächsten Nekropolgebieten von Allianoi gewesen war. Obwohl eine Vielzahl von verschiedenen Grabarten in der näheren Umgebung von Allianoi beobachtet werden konnte, konnten weiteren beschrifteten Grabstelen bei den Notgrabungen nicht aufgefunden werden.

\section{ABKÜRZUNGEN und QUELLEN}

Adak, M., \& Akyürek-Şahin, N. (2005). “Katalog der Inschriften im Museum von Adapazarı”. Gephyra, $2,133-172$.

Akyol, A. (2003). Zaman Tünelinde Yalova. İstanbul.

Atalay, E. (1988). Hellenistik Çăg'da Ephesos Mezar Stelleri Atölyeleri. İzmir.

Börker, C., \& Merkelbach, R. (1979). Die Inschriften von Ephesos. Teil 2, Inschriften griechischer Städte aus Kleinasien 12. Bonn.

Corsten, Th. (1987). Die Inschriften von Apameia (Bithynien) und Pylai. Inschriften griechischer Städte aus Kleinasien 32. Bonn.

Cremer, M. (1991). Hellenistisch-römische Grabstelen im nordwestlichen Kleinasien, 1. Mysien. Asia Minor Studien 4. Bonn.

Debord, P. (1982). Aspects sociaux et économiques de la vie religieuse dans l'Anatolie Gréco-Romaine. Leiden.

Engelmann, H. (2000). "Neue Inschriften aus Ephesos". JÖAI, 69, 77-93.

Fraser, P. M., \& Matthews, E. (2000). A Lexicon of Greek Personal Names. Vol. IIIB. Oxford.

Günther, L. M. (1998). "Das Hochwasser bei Helenopolis (6. Jh.n. Chr.)". Hrg. E. Olshausen, H. Sonnabend. Naturkatastrophen in der antiken Welt, Stuttgarter Kolloquium zur Historischen Geographie des Altertums 6,105-117, 1996. Stuttgart,

Hermann, P., \& Malay, H. (2007). New Documents from Lydia. Wien.

İçten, Ç., \& Engelmann, H. (1998). "Inschriften aus Ephesos und Kolophon”. ZPE, 120, 83-91.

Lajtar, A., \& Petzl, G. (2003). "Eine lydische Familie aus der zweiten Hälfte des 3. Jahrhunderts n. Chr. 
(zu Epigr. Anatol. 34, 2002, 99 ff., Nr. 3 und IGR IV 621)”. EA, 36, 45-50.

Liddell, H. G., \& Scott, R. (1968). Lexicon. A Supplement. Oxford.

Mägele, S. (2005). "Ein besonderer Ort für Votive: Anmerkungen zu einem ungewöhnlichen Befund an drei Statuen aus einem Nymphäum in Sagalassos". MDAI (I), 55, 289-307.

Malay, H. (2003). “A Praise on Men Artemidorou Axiottenos”. EA, 36, 13-18.

Mansel, A. M. (1936). Yalova ve Civarl/Yalova und Umgebung. İstanbul.

Marek, Chr. (2010). Geschichte Kleinasiens in der Antike. München.

Meyer, M. W. (1987). The Ancient Mysteries. A Source Book of Sacred Texts. Pennsylvania.

Mitropoulou, E. (1975). Libation Scenes with Oinochoe in Votive Reliefs. Athens.

Müler, H. (2004). “Allianoi Zur Identifizierung eines antiken Kurbades im Hinterland von Pergamon”. Ist. Mitt, 54, 215-225.

Petzl, G. (1982). Die Inschriften von Smyrna Teil I, Inschriften griechischer Städte aus Kleinasien, 23. Bonn.

Petzl, G. (1987). Die Inschriften von Smyrna Teil 2,1, Inschriften griechischer Städte aus Kleinasien, 24, 1. Bonn.

Pfuhl, E., \& Möbius, H. (1977). Die ostgriechischen Grabreliefs Tafelband I. Mainz am Rhein.

Pfuhl, E., \& Möbius, H. (1979). Die ostgriechischen Grabreliefs Tafelband II. Mainz am Rhein.

Robert, L. (1963). Noms Indigènes dans L’Asie-Mineure Gréco-Romaine. Paris.

Robert, L. (1955). "Dedicaces et Reliefs Votifs. 14. Meter Thermene”. Hellenica, 10, 78-82.

Schwertheim, E. (1987). Die Inschriften von Hadrianoi und Hadrianeia, Inschriften griechischer Städte aus Kleinasien, 33. Bonn.

Sundwall, J. (1913). Die einheimischen Namen der Lykier nebst einem Verzeichnisse kleinasiatischer Namenstämme. Leipzig.

Yaraş, A. (2004). "Die Koca Koru Tepe-Nekropole-Nekropole bei Bergama”. Ist. Mitt., 54, 227-235.

Yaraş, A. (2004-2005). "Die Therme Allianoi”. AA, 2004-2005, 71-74.

Yaraş, A. (2006). "Wasser in der Heiltherme von Allianoi". Hrg. G. Wiplinger, Cura Aquarum in Ephesos, XII, 453-462.

Yaraş, A. (2006). “Allianoi”. Byzas, 3, 19-36.

Yaraş, A., \& Erten, E. (2008). “Allianoi und zwei neue Inschriften über den Kult von Asklepios”. Hrg. E. Schwertheim, Asia Minor Studien 55, Studien zum antiken Kleinasien, VI, 83-91. Bonn.

Yegül, F. (1995). Baths and Bathing in Classical Antiquity. New York.

Zgusta, L. (1964). Kleinasiatische Personennamen. Prag. 